\title{
Total Synthesis of PDE-II
}

\author{
Nobuo Komoto, Yuji Enomoto, Michihiro MiYaGaKI, Yoshinori TanaKa, \\ Kiyoaki NitanaI and Hamao UMEZAwA* \\ Central Research Laboratory, Mitsui Toatsu Chemicals, Inc., \\ 1190, Kasama-cho, Totsuka-ku, Yokohama, Japan \\ *Institute of Microbial Chemistry, \\ 14-23, Kamiosaki 3-chome, Shinagawa-ku, Tokyo, Japan
}

Received August 28, 1978

\begin{abstract}
Starting from 7-hydroxy-6-methoxy-indole (III) PDE-II (II), a new inhibitor of cyclic adenosine $3^{\prime}, 5^{\prime}$-monophosphate phosphodiesterase and the first substance which has 1,2dihydro-3H-pyrrolo[3,2-e]indole skelton, was synthesized in eight steps. The key intermediate, 1-acetyl-5-amino-2,3-dihydro-7-hydroxy-6-methoxy-indole (VIII), carring necessary substituents was obtained from III in five steps. Coupling of the diazoniumsalt of VIII with ethyl 2-acetylpropionate followed by acid catalized cyclization and alkaline hydrolysis afforded II.
\end{abstract}

Recently Enomoto et al. ${ }^{11}$ isolated PDE-I and PDE-II as new inhibitors of cyclic adenosine-3', $5^{\prime}$-monophosphate phosphodiesterase from culture filtrate of streptomyces. They also determined the structures of PDE-I and PDE-II as I and II respectively by a combination of magnetic resonance and X-ray spectroscopic techniques. ${ }^{2)}$ The syntheses of the two inhibitors are interesting from the viewpoint of their new tricyclic molecular structures having 1,2-dihydro-3H-pyrrolo[3,2-e]indole skelton and their biological activities. This paper describes the first total synthesis of PDEII, and we will report the synthesis of PDE-I in the successive paper.

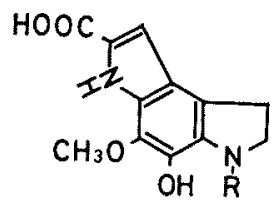

FIG. 1. I: $\mathrm{R}=\mathrm{CONH}_{2}$ PDE-I

II: $\mathrm{R}=\mathrm{COCH}_{3}$ PDE-II

Our initial synthetic target was the hydroindole (VIII) shown in Fig. 2. This key intermediate has the necessary hydroindole skelton and the sites for the introduction of pyrrole moiety of II. The preparation of VIII was carried out as shown in Fig. 2 starting from
III $^{3)}$ which is obtainable in five steps of reactions from vanilline. Hydrogenation of III in the presence of platinum charcoal in acetic acid gave IV. Because of its instability IV was acetylated without any purification to afford V. The resulting bisacetate (V) was then nitrated with fuming nitric acid in acetic anhydride to give VI. Treatment of VI with sodium hydroxide in ethanol at $10^{\circ} \mathrm{C}$ resulted in the formation of the partially deacetylated compound (VII), which was subjected to catalytic hydrogenation using $10 \%$ palladium charcoal in ethanol to afford VIII. We chose JappKlingemann reaction ${ }^{4}$ as the next stage of reaction, because it seemed most straightforward for the preparation of an important intermediate (IX) from VIII. Thus, VIII was converted into the corresponding diazonium salt and treated with ethyl 2-acetylpropionate under neutral conditions to give IX in high yield. Although the hydrazone (IX) showed to be a mixture of syn and anti isomers, $\left.{ }^{5}\right)$ we did not need to separate these isomers because their configurational difference was lost by cyclization at the next stage. According to the Fischer's method, IX was treated with acid to give the ethylester of PDE-II (X). Finally, treatment of the ester $(\mathrm{X})$ with diluted potassium hydroxide solution at room temperature 

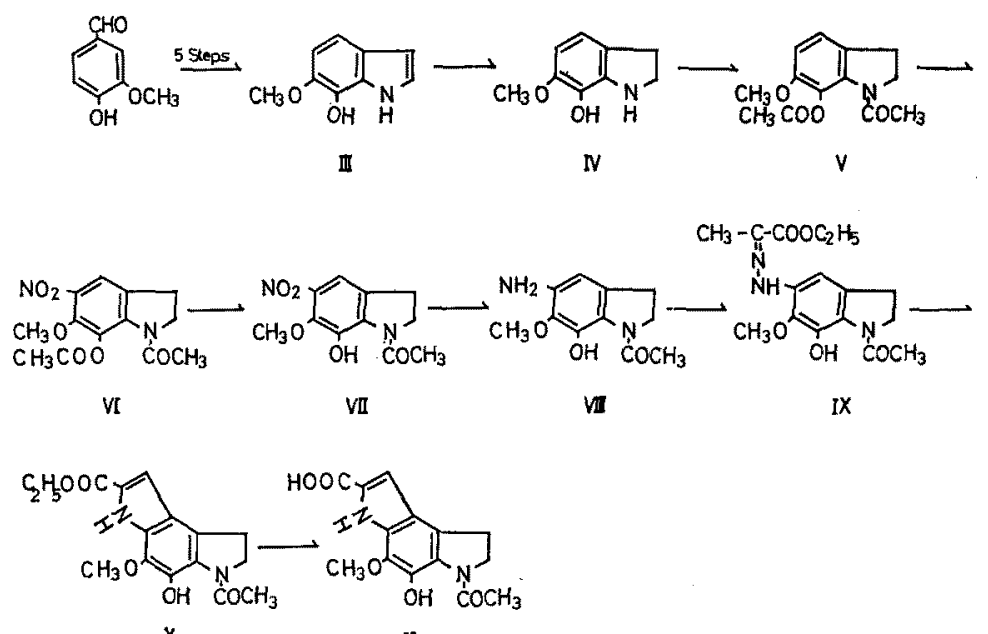

FIG. 2. Synthetic Route of PDE-II.

gave PDE-II (II). The synthetic compound showed the IR and NMR data identical with those of the authentic sample. Further, the melting point of the natural PDE-II showed no depression on admixture with the synthetic sample.

\section{EXPERIMENTAL}

All melting points are uncorrected. Infrared spectra were recorded on a JASCO IRA-I grating infrared spectrophotometer. Proton magnetic resonance spectra were determined on JEOL $3 \mathrm{H}-60(60 \mathrm{MHz})$ nuclear magnetic resonance spectrometer using TMS as an internal standard.

\section{7-Acetoxy-1-acetyl-2,3-dihydro-6-methoxy indole ( V)}

In an autoclave, a solution of III $(20.0 \mathrm{~g})$ in acetic acid $(100 \mathrm{ml})$ in the presence of $5 \% \mathrm{Pt} / \mathrm{C}(10.0 \mathrm{~g})$ was stirred under the hydrogen pressure at $30 \mathrm{~kg} / \mathrm{cm}^{2}$. When hydrogen was no longer absorbed the catalyst was removed by filtration. The filtrate was condensed to yield $12.2 \mathrm{~g}$ of crude acetic acid salt of IV. A mixture consisting of the salt $(4.1 \mathrm{~g})$, pyridine $(10 \mathrm{ml})$ and acetic anhydride $(10 \mathrm{ml})$ was heated at $60^{\circ} \mathrm{C}$ for $3 \mathrm{hr}$, followed by addition of water to decompose excess acetic anhydride. The mixture was concentrated and the residue was recrystallized from carbon tetrachloride.

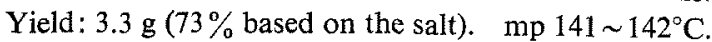
$\mathrm{IR} \nu_{\max }^{\mathrm{KBr}} \mathrm{cm}^{-1}: 1770,1675$. NMR $\delta_{\mathrm{Me}}^{\mathrm{CDCls}}: 2.24(3 \mathrm{H}$, $\left.\mathrm{s}, \mathrm{COCH}_{3}\right), 2.32\left(3 \mathrm{H}, \mathrm{s}, \mathrm{COCH}_{3}\right), 3.01(2 \mathrm{H}, \mathrm{t}, J=$ $\left.7.5 \mathrm{~Hz},-\mathrm{CH}_{2}-\right), 3.82\left(3 \mathrm{H}, \mathrm{s}, \mathrm{OCH}_{\mathrm{s}}\right), 4.13(2 \mathrm{H}, \mathrm{t}, J=$ $\left.7.5 \mathrm{~Hz},-\mathrm{CH}_{2}-\right), 6.70(1 \mathrm{H}, \mathrm{d}, J=8.3 \mathrm{~Hz}, \mathrm{Ar}-\mathrm{H}), 7.07$ $(1 \mathrm{H}, \mathrm{d}, J=8.3 \mathrm{~Hz}, \mathrm{Ar}-\mathrm{H})$. Anal. Found: C, 62.16 ;
$\mathrm{H}, 5.84 ; \mathrm{N}, 5.78$. Calcd. for $\mathrm{C}_{13} \mathrm{H}_{18} \mathrm{NO}_{4}: \mathrm{C}, 62.64$; $\mathrm{H}, 6.07$; N, $5.62 \%$.

\section{7-Acetoxy-1-acetyl-2,3-dihydro-6-methoxy-5-nitro indole} $(V I)$

To a suspension of $\mathrm{V}(5.5 \mathrm{~g})$ in acetic anhydride $(50 \mathrm{ml})$ was added fuming nitric acid $(1.5 \mathrm{ml})$ at $0 \sim 2^{\circ} \mathrm{C}$. After stirring for $1 \mathrm{hr}$ at the temperature the reaction mixture was poured into ice-water. The separated crystals were collected and recrystallized from methanol. Yield: $4.0 \mathrm{~g}(68 \%) . \operatorname{mp} 161 \sim 162^{\circ} \mathrm{C} . \quad$ IR $\nu_{\max }^{\mathrm{KBr}} \mathrm{cm}^{-1}$ : $1770,1680,1515,1460,1380,1340 . \quad$ NMR $\delta_{\text {Mesi }}^{\mathrm{CDCls}}$ : $2.27\left(3 \mathrm{H}, \mathrm{s}, \mathrm{COCH}_{3}\right), 2.35\left(3 \mathrm{H}, \mathrm{s}, \mathrm{COCH}_{3}\right), 3.16(2 \mathrm{H}$, $\left.\mathrm{t}, J=7.5 \mathrm{~Hz},-\mathrm{CH}_{2}-\right), 3.97\left(3 \mathrm{H}, \mathrm{s}, \mathrm{OCH}_{3}\right), 4.20(2 \mathrm{H}, \mathrm{t}$, $\left.J=7.5 \mathrm{~Hz},-\mathrm{CH}_{2}-\right), 7.71(1 \mathrm{H}, \mathrm{s}, \mathrm{Ar}-\mathrm{H})$. Anal. Found: C, 53.47; $\mathrm{H}, 4.67 ; \mathrm{N}, 10.33$. Calcd. for $\mathrm{C}_{18} \mathrm{H}_{18} \mathrm{NO}_{4}$ : C, $53.06 ; \mathrm{H}, 4.80 ; \mathrm{N}, 9.52 \%$.

\section{1-Acetyl-2,3-dihydro-7-hydroxy-6-methoxy-5-nitro indole (VII)}

To a suspension of VI $(3.7 \mathrm{~g})$ in ethanol $(130 \mathrm{ml})$ was added $2 \mathrm{~N}$ sodium hydroxide solution $(40 \mathrm{ml})$ at $10^{\circ} \mathrm{C}$. The resulting mixture was left for $30 \mathrm{~min}$ at the temperature and diluted with water $(200 \mathrm{ml})$ and acidified with diluted hydrochloric acid. The precipitated crystals were collected and recrystallized from methanol. Yield: $2.6 \mathrm{~g}(82 \%) . \mathrm{mp} 146^{\circ} \mathrm{C}$. IR $\nu_{\max }^{\mathrm{KBr}} \mathrm{cm}^{-1}: 2440,1630$, 1600, 1560. NMR $\delta_{\mathrm{Mesi}}^{\mathrm{CDCl}}: 2.36\left(3 \mathrm{H}, \mathrm{s}, \mathrm{COCH}_{3}\right), 3.17$ $\left(2 \mathrm{H}, \mathrm{t}, J=8.0 \mathrm{~Hz},-\mathrm{CH}_{2}-\right), 4.02\left(3 \mathrm{H}, \mathrm{s}, \mathrm{OCH}_{8}\right), 4.19$ $\left(2 \mathrm{H}, \mathrm{t}, J=8.0 \mathrm{~Hz},-\mathrm{CH}_{2}-\right), 7.18(1 \mathrm{H}, \mathrm{s}, \mathrm{Ar}-\mathrm{H}), 12.02$ $(1 \mathrm{H}, \mathrm{s}, \mathrm{OH})$, Anal. Found: C, 52.74; H, $4.71 ; \mathrm{N}, 11.44$. Calcd. for $\mathrm{C}_{11} \mathrm{H}_{12} \mathrm{~N}_{2} \mathrm{O}_{6}: \mathrm{C}, 52.38 ; \mathrm{H}, 4.80 ; \mathrm{N}, 11.11 \%$.

\section{1-Acetyl-5-amino-2,3-dihydro-7-hydroxy-6-methoxy in- dole (VIII)}

In a $200 \mathrm{ml}$-volume of autoclave a solution of VIl 
$(3.7 \mathrm{~g})$ and ethanol $(100 \mathrm{ml})$ stirred with $10 \% \mathrm{Pd} / \mathrm{C}$ $(1.2 \mathrm{~g})$ under the hydrogen pressure at $13 \mathrm{~kg} / \mathrm{cm}^{2}$ until no more hydrogen was absorbed. The catalyst was filtered off. The filtrate was evaporated to dryness and recrystallized from ethanol. Yield: $3.2 \mathrm{~g}$. mp 174 $175^{\circ} \mathrm{C} . \quad$ IR $\nu_{\max }^{\mathrm{KBr}} \mathrm{cm}^{-1}: 3420,3340,2480 \sim 2560$ (broad), 1635. NMR $\delta_{\mathrm{Me}_{4} \mathrm{Si}}^{\mathrm{Acet}-\mathrm{d}_{6}}: 2.24\left(3 \mathrm{H}, \mathrm{s}, \mathrm{COCH}_{3}\right), 2.98$ $\left(2 \mathrm{H}, \mathrm{t}, J=8.0 \mathrm{~Hz},-\mathrm{CH}_{2}-\right), 3.76\left(3 \mathrm{H}, \mathrm{s}, \mathrm{OCH}_{3}\right), 4.11$ $\left(2 \mathrm{H}, \mathrm{t}, J=8.0 \mathrm{~Hz},-\mathrm{CH}_{2}-\right), 3.90 \sim 4.60(2 \mathrm{H}$, broad s, $\left.\mathrm{NH}_{2}\right), 6.16(1 \mathrm{H}, \mathrm{s}, \mathrm{Ar}-\mathrm{H}), 12.18(1 \mathrm{H}, \mathrm{s}, \mathrm{OH})$. Anal. Found: $\mathrm{C}, 59.69 ; \mathrm{H}, 6.41 ; \mathrm{N}, 12.56$. Calcd. for $\mathrm{C}_{11} \mathrm{H}_{14} \mathrm{~N}_{2} \mathrm{O}_{3}: \mathrm{C}, 59.45 ; \mathrm{H}, 6.35 ; \mathrm{N}, 12.60 \%$.

\section{1-Acetyl-2,3-dihydro-5-[N'(1-ethoxycarbonylethylidene) hydrazino]7-hydroxy-6-methoxy indole (IX)}

A cold $\left(0^{\circ} \mathrm{C}\right)$ solution of VIII $(3.0 \mathrm{~g})$ dissolved in a mixture of concentrated hydrochloric acid $(10.4 \mathrm{ml})$ and water $(20 \mathrm{ml})$ was diazotized by addition of a sat. solution of sodium nitrite $(1.1 \mathrm{~g})$. This solution was kept at $-5 \sim 0^{\circ} \mathrm{C}$ while ethyl 2-acetylpropionate $(2.5 \mathrm{~g})$ in ethanol $(31 \mathrm{ml})$ was mixed at $-5 \sim 5^{\circ} \mathrm{C}$ with a solution of sodium hydroxide $(5.0 \mathrm{~g})$ in water $(74 \mathrm{ml})$. Then the preserved diazonium solution was added to it at $0^{\circ} \mathrm{C}$ with stirring. After stirring for $1 \mathrm{hr}$ at $0^{\circ} \mathrm{C}$, the precipitated product was collected, treated with charcoal in ethyl acetate, dricd over anhydrous magnesium sulfate, freed from the solvent in vacuo and recrystallized from ethanol. Yield: $4.3 \mathrm{~g}(95 \%) . \mathrm{mp}$ $194 \sim 195^{\circ} \mathrm{C}$. Anal. Found: C, 57.52; H, 6.09; N, 13.19 . Calcd. for $\mathrm{C}_{18} \mathrm{H}_{20} \mathrm{~N}_{3} \mathrm{O}_{5}: \mathrm{C}, 57.50 ; \mathrm{H}, 6.31 ; \mathrm{N}, 12.53 \%$.

Ethyl 3-acetyl-1,2-dihydro-4-hydroxy-5-methoxy-3H-pyrrolo[3,2,-e]indole-7-carboxylate $(X)$

i) Cyclization with hydrochloric acid. Through a suspension of IX $(0.80 \mathrm{~g})$ in ethanol $(50 \mathrm{ml})$ was passed dry hydrogen chloride gas at $5 \sim 10^{\circ} \mathrm{C}$ for $90 \mathrm{~min}$. The reaction mixture was poured into ice-water and extracted with chloroform-methanol $(50: 1)$ followed by recrystallization from ethanol. Yield: $0.08 \mathrm{~g}(11 \%)$. mp 241 242 $\sim$ C. IR $\nu_{\max }^{\mathrm{KBr}} \mathrm{cm}^{-1}: 3320, \quad 2460 \sim 2340$

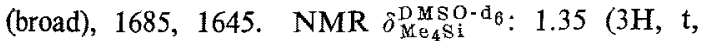
$\left.J=7.5 \mathrm{~Hz}, \mathrm{CH}_{3}\right), 2.29\left(3 \mathrm{H}, \mathrm{s}, \mathrm{COCH}_{3}\right), 3.20(2 \mathrm{H}, \mathrm{t}$, $\left.J=8.0 \mathrm{~Hz},-\mathrm{CH}_{2}-\right), 3.84\left(3 \mathrm{H}, \mathrm{s}, \mathrm{OCH}_{3}\right), 4.25(2 \mathrm{H}, \mathrm{t}$, $\left.J=8.0 \mathrm{~Hz},-\mathrm{CH}_{2}-\right), 4.28\left(2 \mathrm{H}, \mathrm{q}, J=7.5 \mathrm{~Hz}, \mathrm{O}-\mathrm{CH}_{2}-\right.$ Me), $7.06(1 \mathrm{H}, \mathrm{d}, J=2.0 \mathrm{~Hz}$, indolic $\mathrm{H}), 11.65(1 \mathrm{H}, \mathrm{s}$, $\mathrm{NH}), 12.34(1 \mathrm{H}, \mathrm{s}, \mathrm{OH})$. Anal. Found: $\mathrm{C}, 60.17 ; \mathrm{H}$, $5.73 ; \mathrm{N}, 8.55$. Calcd. for $\mathrm{C}_{18} \mathrm{H}_{18} \mathrm{~N}_{2} \mathrm{O}_{5}: \mathrm{C}, 60.37 ; \mathrm{H}$, $5.70 ; \mathrm{N}, 8.80 \%$. ii) Cyclization with sulfuric acid. To a suspension of IX $(4.50 \mathrm{~g})$ in ethanol $(45 \mathrm{ml})$ was added concentrated sulfuric acid $(9 \mathrm{ml})$ and the reaction mixture was heated at $65 \sim 70^{\circ} \mathrm{C}$ for $70 \mathrm{~min}$. The reaction mixture was worked as above. Yield: $0.78 \mathrm{~g}(18 \%)$.

\section{3-Acetyl-1,2-dihydro-4-hydroxy-5-methoxy-3H-pyrrolo}

[3,2-e]indole-7-carboxylic acid (II)

To a suspension of $X(200 \mathrm{mg})$ in ethanol $(10 \mathrm{ml})$ was added $1 \mathrm{~N}$ potassium hydroxide solution $(13 \mathrm{ml})$. The mixture was stirred for $90 \mathrm{~min}$ at room temperature, diluted with water $(100 \mathrm{ml})$ and acidified with diluted hydrochloric acid. The aqueous solution was extracted with ethyl acetate and the organic layer was dried over anhydrous sodium sulfate and concentrated. The residue was purified with preparative thin-layer chromatography using silicic acid (Merck $\mathrm{GF}_{254}$ ). Development with chloroform-methanol (2:1) afforded II. Yield: $70 \mathrm{mg}(38 \%) \cdot \mathrm{mp} 249^{\circ} \mathrm{C}$ (dec.). IR $2_{\mathrm{max}}^{\mathrm{KBr}} \mathrm{cm}^{-1}$ : $3300,1670,1640 . \quad$ NMR $\tilde{o}_{\mathrm{Me}_{4} \mathrm{Si}}^{\mathrm{DMSO}-\mathrm{d}_{6}}: 2.28(3 \mathrm{H}, \mathrm{s}$, $\left.\mathrm{COCH}_{3}\right), 3.20\left(2 \mathrm{H}, \mathrm{t}, J=8.0 \mathrm{~Hz},-\mathrm{CH}_{2}-\right), 3.79(3 \mathrm{H}, \mathrm{s}$, $\left.\mathrm{OCH}_{3}\right), 4.22\left(2 \mathrm{H}, \mathrm{t}, J=8.0 \mathrm{~Hz},-\mathrm{CH}_{2}-\right), 6.96(1 \mathrm{H}, \mathrm{d}$, $J=2.0 \mathrm{~Hz}$, indolic $\mathrm{H}), 11.41(1 \mathrm{H}, \mathrm{s}, \mathrm{NH}), 12.18(1 \mathrm{H}, \mathrm{s}$, $\mathrm{OH}$ ). Anal. Found: C, 57.71; H, 4.78; N, 9.50. Calcd. for $\mathrm{C}_{14} \mathrm{H}_{14} \mathrm{~N}_{2} \mathrm{O}_{8}: \mathrm{C}, 57.93 ; \mathrm{H}, 4.86 ; \mathrm{N}, 9.65 \%$.

Acknowledgements. The authors express their sincere thanks to Dr. E. Otsuka, the Exective Managing Director of Mitsui Toatsu Chemicals Inc., and Mr. S. Yoshioka, the Director of Mitsui Toatsu Central Research Laboratory, for their encouragement throughout this research.

\section{REFERENCES}

1) Y. Enomoto, Y. Furutani, H. Naganawa, $M$. Hamada, T. Takeuchi and H. Umezawa, Agric. Biol. Chem., 42, 1331 (1978).

2) H. Nakamura, Y. Enomoto, T. Takeuchi, H. Umezawa and Y. Iitaka, ibid., 42, 1337 (1978).

3) R. J. S. Beer, K. Clarke, H. F. Davenport and A. Robertson, J. Chem. Soc., 1951, 2037.

4) R. R. Phillips, "Org. Reaction," Vol.10, ed. by R. Adams, John Willey and Sons, Inc., New York, N. Y., 1959, p. 143.

5) H. Henecka, H. Timmer, R. Lorenz and W. Geiger, Chem. Ber., 90, 1060 (1957). 